\title{
Morphological changes in the meibomian glands of patients with phlyctenular keratitis: a multicenter cross-sectional study
}

\author{
Takashi Suzuki ${ }^{1,2^{*}}$, Naoyuki Morishige ${ }^{1,3}$, Reiko Arita ${ }^{1,4,5}$, Shizuka Koh ${ }^{1,6}$, Tohru Sakimoto ${ }^{1,7}$, Rika Shirakawa ${ }^{1,4}$, \\ Kazunori Miyata ${ }^{8}$ and Yuichi Ohashi ${ }^{2}$
}

\begin{abstract}
Background: Phlyctenular keratitis is a hypersensitivity reaction of the cornea, and a complication of eyelid margin disease in children and young adults. In this study, we compared the morphology of the meibomian glands in eyelids between phlyctenular keratitis patients and healthy young adults, using noncontact meibography.

Methods: The study included 16 eyes of 13 patients diagnosed with phlyctenular keratitis and 17 eyes of 17 healthy volunteers. Slit-lamp observations of the cornea and eyelid were performed on all subjects. The morphology of the meibomian glands was scored using non-contact meibography (meiboscore). The meiboscore in worse eye was used in bilateral phlyctenular keratitis.

Results: All eyes with phlyctenular keratitis, but not normal controls, showed corneal nodules, neovascularization, and superficial punctate keratopathy. The mean meiboscore in phlyctenular keratitis patients (upper lid: $2.9 \pm 0.3$, lower lid: $2.7 \pm 0.5$ ) was significantly higher than in controls (upper lid: $0.4 \pm 0.6$, lower lid: $0.1 \pm 0.3$ ).

Conclusions: Noncontact meibography enabled visualization of meibomian gland loss in phlyctenular keratitis patients, suggesting a relationship between abnormalities of the meibomian glands in young individuals and the pathogenesis of phlyctenular keratitis.
\end{abstract}

Keywords: Phlyctenular keratitis, Meibomian glands, Meibography, Meibomitis

\section{Background}

Phlyctenular keratitis is a complication of eyelid margin disease, primarily affecting children and young adults [1, 2]. The characteristic clinical findings in phlyctenular keratitis include inflammatory corneal nodules with vascularization and meibomitis [3]. The pathogenesis of this disease could involve a delayed-type hypersensitivity (DTH) reaction to foreign microbial proteins from organisms such as Mycobacterium tuberculosis, Staphylococcus aureus, or Propionibacterium acnes, which are found at the eyelid margin and in the meibomian gland [3-10]. Indeed, phlyctenular keratitis can be accompanied by meibomitis or blepharitis, and bacteria such as S. aureus and P. acnes can

\footnotetext{
* Correspondence: t-suzuki@m.ehime-u.ac.jp

${ }^{1}$ LIME (Lid and Meibomian gland) working group, Tokyo, Japan

2Department of Ophthalmology, Ehime University, Graduate School of

Medicine, Shitsukawa, Toon, Ehime 791-0295, Japan

Full list of author information is available at the end of the article
}

be detected in eyelid scrapings or in meibum $[3,4,10]$. Meibomitis, which is inflammation of the meibomian glands, causes the glands to be obstructed by thick waxy secretions, and may trigger phlyctenular keratitis [3]. Suzuki et al. proposed a disease subset termed meibomitis-related keratoconjunctivitis (MRKC), ocular surface inflammatory disease associated with meibomitis; the clinical features of MRKC are similar or identical to those of phlyctenular keratitis [7]. Thus, the observation of eyelid and meibomian gland condition in cases of phlyctenular keratitis may help us to understand the pathogenesis of the disease.

Meibomian gland condition is commonly evaluated based on slit-lamp observations of the meibomian orifices, examination of meibomian gland sebum, and meibography, which assesses the structure of the glands by transillumination. However, it is often difficult to observe the condition of the eyelid or of the meibomian glands in children who cannot be secured to a chin-rest. 
Additionally, conventional meibography and a slit lamp allows observation of only a limited area of the eyelid [11]. Recently, a noninvasive, mobile, pen-shaped meibography system using an infrared light-emitting diode was developed, and was found to be useful in the observation of meibomian gland structure in infants and in patients with severe systemic diseases [12-14].

Because meibomitis is associated with phlyctenular keratitis, abnormalities of the meibomian glands may be involved its pathogenesis. Little is known, however, about the morphology of the meibomian glands in this disease. Herein we report features of patients with phlyctenular keratitis, observed with noncontact meibography, and compare them to those of healthy volunteers. These observations demonstrate the relationship of morphological changes in meibomian glands to phlyctenular keratitis in patients, and suggest its pathogenesis.

\section{Methods}

\section{Patients}

Patients with phlyctenular keratitis and healthy young adults were examined in Ehime University hospital, Yamaguchi University Hospital, Itoh Clinic, Osaka University Hospital, Nihon University Hospital, Miyata Eye Hospital, and Tokyo University Hospital. Diagnosis of phlyctenular keratitis was made based on bacterial culture of eye lids and clinical manifestations such as corneal nodules, their vascularization, and meibomitis, as described previously [3]. Subjects included 13 patients (four males and nine females; mean \pm SD age, $13.8 \pm$ 8.6 years) and 17 healthy volunteers (seven males and ten females; mean \pm SD age, $13.4 \pm 2.7$ years).

\section{Examination}

Slit-lamp observations of the cornea and eyelid were performed. The upper and lower eyelids were turned over and the meibomian glands were observed using a mobile pen-shaped meibograph (Meibopen, JFC Sales Plan Co. Ltd, Tokyo, Japan) or another non-contact meibography system (BG-4 M, TOPCON, Tokyo, Japan). Captured pictures were estimated by graders who were masked to the demographics of the subjects. Partial or complete loss of the meibomian glands for each eyelid was graded by meiboscore as reported previously; grade 0 (no loss of meibomian glands), grade 1 (loss of $1 / 3$ of the total area of meibomian glands), grade 2 (area loss between $1 / 3$ and 2/ 3 ), and grade 3 (area loss $\geq 2 / 3$ ) [15]. Meiboscores for the upper and lower eyelids were summed to obtain a score for each eye [15]. The meiboscore in worse eye was used in bilateral phlyctenular keratitis.

\section{Statistical analysis}

Meiboscores and ages were compared between phlyctenular keratitis patients and healthy volunteers using Mann-
Whitney $U$-test (JMP pro 11, SAS Institute Inc., NC). In cases of unilateral phlyctenular keratitis, we compared the meiboscores between the inflamed and non-inflamed eyes using two-tailed Student's $t$-test. Gender were compared between phlyctenular keratitis patients and healthy volunteers using a chi-square test. A $p$ value of $<0.05$ was considered to indicate statistical significance. Data are shown as means \pm SD unless otherwise specified.

\section{Results}

A representative clinical photograph of phlyctenular keratitis in a 4-year-old male (patient case 9) with a corneal nodule, neovascularization, hyperemia, and meibomitis in the right eye is shown in Fig. 1. Ocular surface manifestations in 13 patients with phlyctenular keratitis are summarized in Table 1 . There were three adults ( $>20$ years), and ten young adults ( $<16$ years). Four of thirteen patients had bilateral phlyctenular keratitis. Inflamed eyes of all patients had corneal nodules, neovascularization, and superficial punctate keratopathy (SPK). Five patients had a history of chalazia. Healthy volunteers showed no clinical features. Gender and age were not significantly different between the phlyctenular keratitis patients and the healthy volunteers (Table 2).

The morphology of the meibomian glands in the eyelids of 13 phlyctenular keratitis patients (13 eyes) and 17 healthy volunteers (17 eyes) were compared. This demonstrated meibomian gland loss in both the upper and lower lids of the right eye of the patient identified as case 9 (Meiboscore;upper lid:3, lower lid:3, total:6), while this patient's non-inflamed left eye showed meibomian glands of a normal morphology (Figs. 2a and b). The meiboscores of the eyelids of phlyctenular keratitis patients and healthy volunteers are summarized in Table 2. Mean meiboscores of both the upper and lower lids with phlyctenular keratitis were significantly higher than those of healthy volunteers. In seven of nine patients with unilateral phlyctenular keratitis, we compared the mean meiboscores of inflamed and non-inflamed eyes. In other two patients with unilateral phlyctenular keratitis, the meibomian glands of non-inflamed eyes were not observed. The mean meiboscore of both the upper and lower lids with phlyctenular keratitis (upper lid: $3.0 \pm 1.7$, lower lid: $2.8 \pm 1.4$ ) was significantly higher than that of noninflamed lids (upper lid: $1.1 \pm 1.1$, lower lid: $1.1 \pm 1.3$ ) $(P<0.05)$. Locations of corneal nodules in phlyctenular keratitis patients were not consistent with the locations of meibomian gland losses. Meibography in patients with phlyctenular keratitis yielded lowcontrast images of meibomian glands, due to conjunctival edema, and the loss of distal meibomian glands from meibomian orifices. 


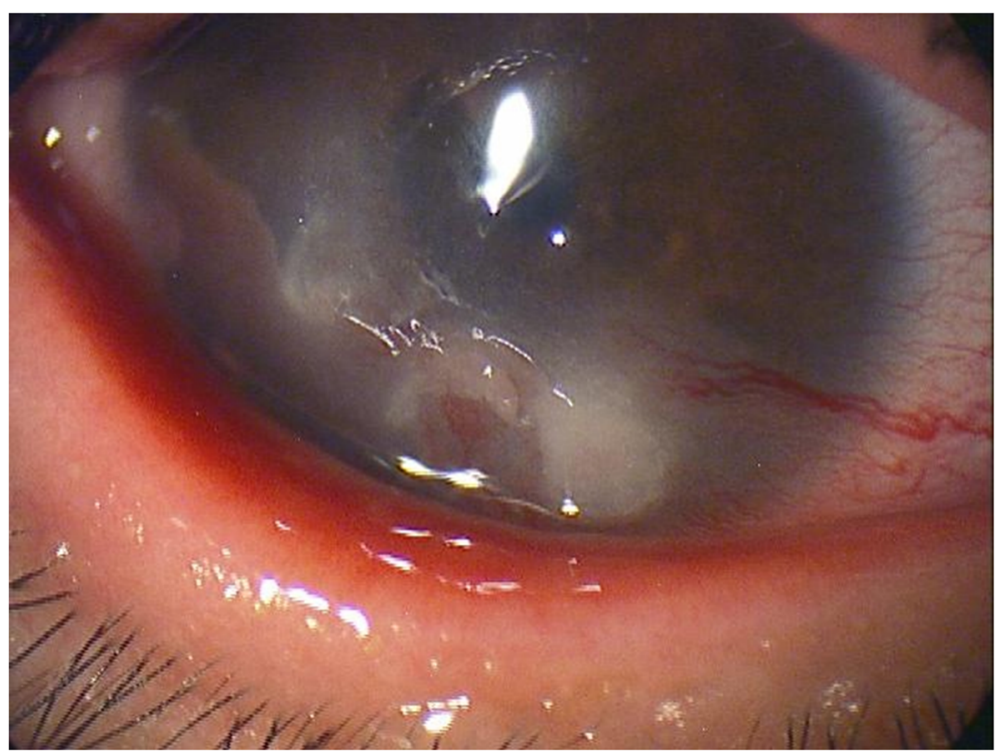

Fig. 1 Slit-lamp photograph of case 9 showing corneal nodule, vascularization, and conjunctival injection

\section{Discussion}

In this study, meibography revealed frequent meibomian gland losses in patients with phlyctenular keratitis, while the morphology of meibomian glands in healthy controls was normal. Moreover meiboscore of lids with phlyctenular keratitis was higher than that of non-inflamed lids in patients with unilateral phlyctenular keratitis. Previous studies have reported a relationship between phlyctenular keratitis and inflammation of the eyelids and meibomian glands [3-10]. Koh et al. demonstrated meibomian gland loss in an eye with unilateral marginal staphylococcal keratitis and eyelid inflammation [16].
Meibomian gland inflammation may induce ocular surface inflammatory disease [7]. Morphology of meibomian gland may be influenced by meibomian gland inflammation because inflammatory cells could obstruct meibomian gland. Thus patients with phlyctenular keratitis may accompany with meibomian gland losses. Moreover there are other possibilities. Since abnormalities of meibomian glands have been found in allergic conjunctivitis and in contact lens wearers [13], meibomian gland loss in phlyctenular keratitis might be caused by the mechanical friction between the ocular surface and eyelid, by inflammation of the cornea and conjunctiva, and

Table 1 Summary of 13 patients with phlyctenular keratitis

\begin{tabular}{|c|c|c|c|c|c|c|c|c|c|c|c|}
\hline \multirow[b]{2}{*}{ Case } & \multirow[b]{2}{*}{ Gender } & \multirow[b]{2}{*}{ Age range of onset (y) } & \multirow[b]{2}{*}{ Eye(s) } & \multirow[b]{2}{*}{ History of chalazia } & \multicolumn{4}{|c|}{ Ocular manifestation } & \multicolumn{3}{|c|}{ Meiboscore } \\
\hline & & & & & Corneal nodule & NV & SPK & Hyperemia & Upper lid & Lower lid & Total \\
\hline 1 & $\mathrm{~F}$ & $20-30$ & OS & + & + & + & + & & 3 & 2 & 5 \\
\hline 2 & $\mathrm{~F}$ & $20-30$ & OS & - & + & + & + & + & 3 & 3 & 6 \\
\hline 3 & $\mathrm{~F}$ & $10-20$ & OU & - & + & + & + & & 3 & 2 & 5 \\
\hline 4 & $\mathrm{~F}$ & $10-20$ & OU & - & + & + & + & + & 3 & 3 & 6 \\
\hline 5 & $\mathrm{~F}$ & $30-40$ & OU & - & + & + & + & + & 3 & 3 & 6 \\
\hline 6 & $\mathrm{~F}$ & $10-20$ & OS & - & + & + & + & + & 2 & 3 & 5 \\
\hline 7 & M & $10-20$ & OD & + & + & + & + & + & 3 & 3 & 6 \\
\hline 8 & $M$ & $0-10$ & OS & + & + & + & + & + & 3 & 3 & 6 \\
\hline 9 & $M$ & $0-10$ & $O D$ & + & + & + & + & + & 3 & 3 & 6 \\
\hline 10 & M & $0-10$ & OS & - & + & + & + & + & 3 & 3 & 6 \\
\hline 11 & $F$ & $10-20$ & OS & + & + & + & + & + & 3 & 3 & 6 \\
\hline 12 & $F$ & $10-20$ & $\mathrm{OD}$ & - & + & + & + & + & 3 & 2 & 5 \\
\hline 13 & $\mathrm{~F}$ & $10-20$ & OU & - & + & + & + & + & 3 & 2 & 5 \\
\hline
\end{tabular}

$F$ female, $M$ male, $O D$ right eye, $O S$ left eye, $O U$ both eyes, $N V$ corneal neovascularization, SPK superficial punctate keratopathy OU showed identical clinical features 
Table 2 Clinical features and meiboscores in patients and controls

\begin{tabular}{llll}
\hline & Phlyctenular keratitis $(n=16)$ & Controls $(n=17)$ & $P$ \\
\hline Gender (M/F) & $4 / 9$ & $7 / 10$ & 0.56 \\
Age & $13.8 \pm 8.6$ & $13.4 \pm 2.7$ & 0.56 \\
Meiboscore (Mean \pm SD) & & \\
Upper & $2.9 \pm 0.3$ & $0.4 \pm 0.6$ & $<0.0001$ \\
Lower & $2.7 \pm 0.5$ & $0.1 \pm 0.3$ & $<0.0001$ \\
\hline
\end{tabular}

$F$ female, $M$ male, $N$ number of patients

bacterial exposure. Another possibility is that meibomitis with meibomian gland loss occurs before the appearance of phlyctenular keratitis, and imbalances in the bacterial flora of meibomian glands, including P. acnes, induce a corneal DTH reaction to a microbial protein, causing phlyctenular keratitis. However some limitations exist in our study. First, since we did not check bacterial culture of eye lids and meibomian gland in all patients, little is known about relationship between bacteria species and morphology of meibomian gland. Along with P. acnes, Staphylococcus aureus which can produce toxins and proteases might be related to pathology of meibomian gland. Second, patients group included three adults ( $>20$ years). Thus ages could influence to meibomian gland pathology. We need to increase number of patients and investigate relationship between ages of patients with phlyctenular keratitis and morphology of meibomian gland. Third, we did not check meibography after treatment for phlyctenular keratitis using antibiotics and steroids. It is important to know if morphology of meibomian gland normalizes after disappearance of inflammation in cornea and meibomian gland. Thus further investigation revealing the relationship between meibomian gland loss and phlyctenular keratitis is required.

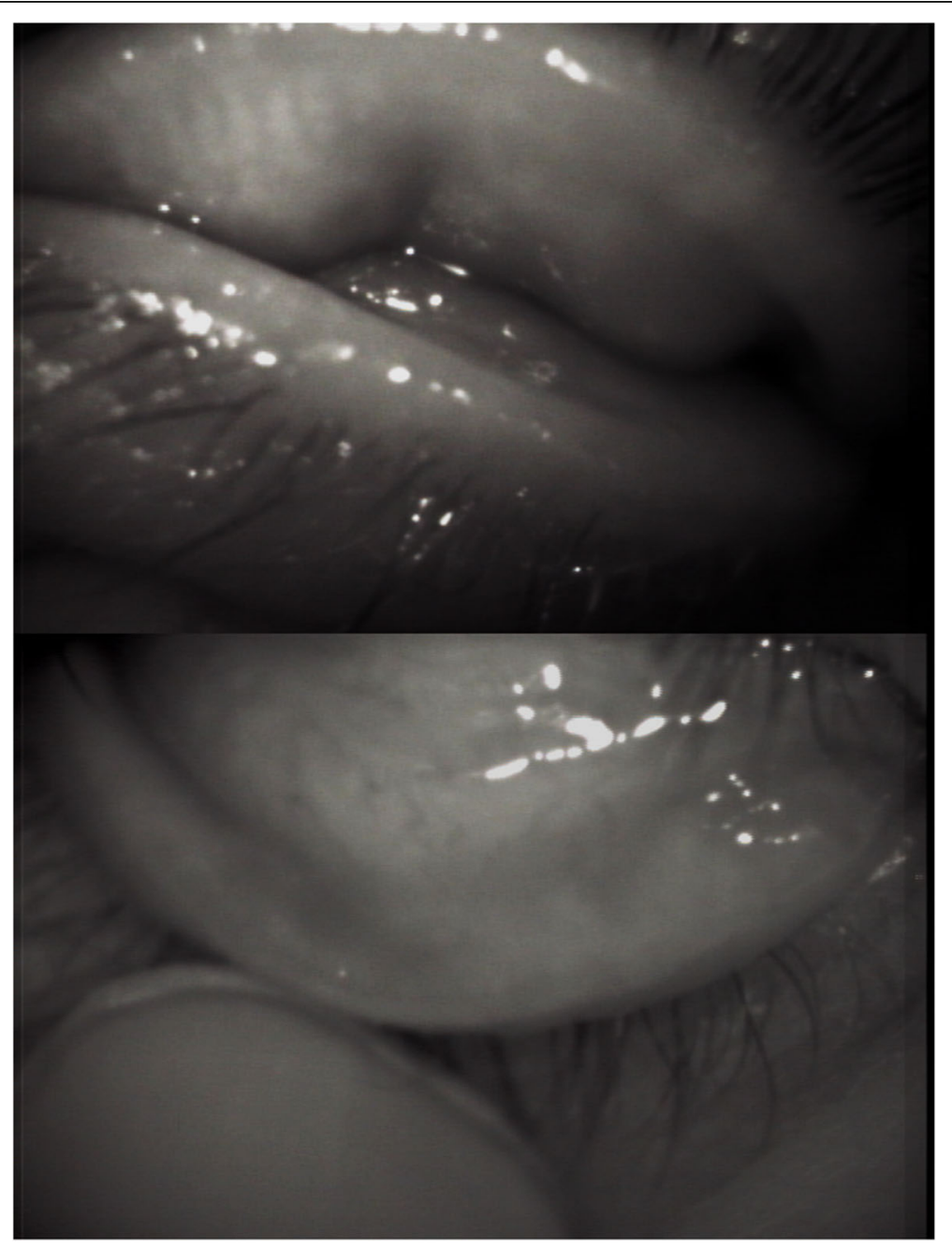

Fig. 2 Noncontact meibography imaging of case 9 showing marked loss of the meibomian glands in the upper (top) and lower eyelids (bottom). Meiboscore (upper lid:3, lower lid:3, total:6) 
The diagnosis of phlyctenular keratitis is made based on clinical manifestations, such as corneal nodules, their vascularization, and meibomitis, along with a positive bacterial culture of the eyelid or meibum. Adding to these conventional examinations, noncontact meibography is useful for visualization of the condition of the meibomian glands. Our data demonstrate a high frequency of meibomian gland loss in phlyctenular patients.

\section{Conclusions}

Noncontact meibography enabled visualization of meibomian gland loss in phlyctenular keratitis patients, indicating that meibomian gland abnormality in young individuals may be related to the pathogenesis of phlyctenular keratitis.

\section{Additional file}

Additional file 1: Summary of 13 patients with phlyctenular keratitis. (XLSX $11 \mathrm{~kb})$

\section{Abbreviations}

DTH: Delayed-type hypersensitivity; MRKC: Meibomitis-related keratoconjunctivitis; SD: Standard deviation; SPK: Superficial punctate keratopathy

\section{Acknowledgements}

None.

\section{Funding}

No author has a financial or proprietary interest in any material or method used in this study.

\section{Availability of data and materials}

Raw data sheet (Additional file 1) has been submitted along with the manuscript.

\section{Authors' contributions}

TS, NM, SK and RA: Conception and design, acquisition, analysis and interpretation of data, drafting of manuscript, administrative and technical support. TS, RS and KM: Analysis and interpretation of data. YO: Supervision. Read and approved the final manuscript. All authors approved the manuscript for submission

\section{Competing interests}

Dr. Arita holds a patent for the meibography technique described in this manuscript. This work did not receive any financial support.

\section{Consent for publication}

Verbal informed consent was obtained from all participants to publish this information, and verbal parental consent to publish was obtained for all patients under 18

\section{Ethics approval and consent to participate}

Verbal informed consent was obtained from all patients and volunteers before examination. For participants whose age was younger than 18 years, verbal informed consent and permission was collected from their parents. This study was approved by the Institutional Review Board of each hospital (Ehime University Hospital, Tokyo University Hospital, Nihon University Hospital, Yamaguchi University Hospital, and Osaka University Hospital), and followed the tenets of the Declaration of Helsinki.

\section{Author details}

'LIME (Lid and Meibomian gland) working group, Tokyo, Japan. ${ }^{2}$ Department of Ophthalmology, Ehime University, Graduate School of Medicine, Shitsukawa, Toon, Ehime 791-0295, Japan. ${ }^{3}$ Department of Ophthalmology, Yamaguchi University, Graduate School of Medicine, Ube, Japan.
${ }^{4}$ Department of Ophthalmology, University of Tokyo School of Medicine, Tokyo, Japan. ${ }^{5}$ Department of Ophthalmology, Itoh Clinic, Saitama, Japan. ${ }^{6}$ Department of Ophthalmology, Osaka University Graduate School of Medicine, Suita, Japan. ${ }^{7}$ Department of Visual Sciences, Division of Ophthalmology, Nihon University School of Medicine, Tokyo, Japan. ${ }^{8}$ Miyata Eye Hospital, Miyakonojo, Japan.

Received: 24 November 2015 Accepted: 15 June 2016

Published online: 10 October 2016

References

1. Mozayeni RM LS. Phylctenular keratoconjunctivitis and marginal staphylococcal keratitis. In: Krachmer JH MM, Holland EJ, editors. Cornea fundamentals, diagnosis and management. Philadelphia: Elsevier Mosby; 2005. p. 1235-40.

2. Robin JB DR, Robin SB. Immunologic disorders of the cornea and conjunctiva. In: Kaufman HE BB, McDonald MB. Woburn, MA, editors. The cornea, 2nd ed. Philadelphia: Butterworth-Heinemann; 1999: p. 581-2.

3. Suzuki T, Mitsuishi Y, Sano Y, Yokoi N, Kinoshita S. Phlyctenular keratitis associated with meibomitis in young patients. Am J Ophthalmol. 2005; 140(1):77-82.

4. Smolin G, Okumoto M. Staphylococcal blepharitis. Arch Ophthalmol. 1977:95(5): 812-6.

5. Sorsby A. The aetiology of phlyctenular ophthalmia. Br J Ophthalmol. 1942;26(5): 189-215.

6. Sorsby A. The aetiology of phlyctenular ophthalmia. Br J Ophthalmol. 1942; 26(4):159-79.

7. Suzuki T. Meibomitis-related keratoconjunctivitis: implications and clinical significance of meibomian gland inflammation. Cornea. 2012;31 Suppl 1:S41-4.

8. Suzuki T, Sano Y, Sasaki O, Kinoshita S. Ocular surface inflammation induced by propionibacterium acnes. Cornea. 2002;21(8):812-7.

9. Thygeson P. The etiology and treatment of phlyctenular keratoconjunctivitis. Am J Ophthalmol. 1951:34(9):1217-36.

10. Thygeson P. Complications of staphylococcic blepharitis. Am J Ophthalmol. 1969;68(3):446-9.

11. Nichols JJ, Berntsen DA, Mitchell GL, Nichols KK. An assessment of grading scales for meibography images. Cornea. 2005;24(4):382-8.

12. Arita R. Validity of noninvasive meibography systems: noncontact meibography equipped with a slit-lamp and a mobile pen-shaped meibograph. Cornea. 2013;32 Suppl 1:S65-70.

13. Arita R, Itoh K, Maeda S, Maeda K, Amano S. A newly developed noninvasive and mobile pen-shaped meibography system. Cornea. 2013;32(3):242-7.

14. Shirakawa R, Arita R, Amano S. Meibomian gland morphology in Japanese infants, children, and adults observed using a mobile pen-shaped infrared meibography device. Am J Ophthalmol. 2013;155(6):1099-103. e1.

15. Arita $\mathrm{R}$, Itoh $\mathrm{K}$, Inoue $\mathrm{K}$, Amano S. Noncontact infrared meibography to document age-related changes of the meibomian glands in a normal population. Ophthalmology. 2008;115(5):911-5.

16. Koh S, Maeda N, Nishida K. Visualization of the meibomian glands by means of noncontact mobile-type meibography (Meibopen) in a 16-year-old girl with unilateral marginal staphylococcal keratitis. J AAPOS. 2014;18(1):99-101.

Submit your next manuscript to BioMed Central and we will help you at every step:

- We accept pre-submission inquiries

- Our selector tool helps you to find the most relevant journal

- We provide round the clock customer support

- Convenient online submission

- Thorough peer review

- Inclusion in PubMed and all major indexing services

- Maximum visibility for your research

Submit your manuscript at www.biomedcentral.com/submit
Biomed Central 